\title{
The hydrostatic equilibrium and its implications on the mechanical properties of planets
}

\author{
P. BALDi - M. CAPUTO (*)
}

Received on April 7th, 1974

SUmmary. - Under the hypothesis of hydrostatic equilibrium the planets of the solar system are represented on the plane $\left(J_{2}, w^{2} a^{3} / M G\right)$ $\left(J_{2}=\right.$ second term of the gravitational potential, $w=$ rotation rate of the planet, $a$-equatorial radius, $M-$ mass of the planet, $G=$ gravitational constant). Since the points representing the planets lie on a straight line it is possible to obtain informations on the giration moment and the moment of inertia of Uranus and Pluto.

Riassunto. - Partendo dall 'ipotesi di equilibrio idrostatico, i pianeti del sistema solare sono rappresentati nel piano $\left(J_{2}, w^{5} a^{3} / M I G\right)$, dove $J_{2}$ è il secondo termine dell'espressione del potenziale gravitazionale, $w$ la velocità di rotazione del pianeta, $a$ il raggio equatoriale, $M I$ la massa del pianeta, $G$ la costante gravitazionale.

Poiché i punti che rappresentano i pianeti giacciono su una linea retta, è possibile avere informazioni sul momento giratore e sul momento di inerzia di Urano e Plutone.

\section{1. - INTRODUCTION.}

Investigations so far carried out on the solar system would seem to indicate a fair degree of regularity in the system itself, and also the presence of special mechanisms $\left(^{5}\right)$ : among these let us recall the regularity of the orbits of the planets (slight inclination, slight eccentricity, anticlock-wise motion, etc.); in the law governing the relation-

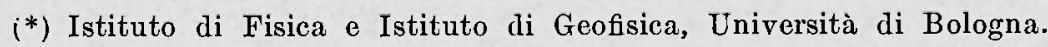


ship of the semimajor axes of two contiguous planets $\left(a_{n+1} j a_{n}-\right.$ const., Titius-Borle law); the alignment of the representative points of the planets in the $C w / M, M$ plane (i).

The study of the motion of artificial and natural satellites makes it possible to obtain the parameters linked to the internal and external structure of the planet such, for example, as the total mass $M$, the flattening at the poles $e, J_{2}$ (the second term of gravitational potential), the moments of inertia.

Considering the planets to be in hydrostatic equilibrium by means of the above-mentioned terms it is possible to arrive, as we shall later show, at a knowledge of the values of $p\left(C / M r^{2}\right)$, and consequently at the value of the moments of inertia of almost all the planets.

\section{2. - THE hydrostatic EqUilibrium of a PLANET.}

Let us consider a planet in hydrostatic equilibrium: approximating in the first order, the equations that link flattening and $J_{2}, m$ $\left(=v^{2} r^{3} / M G\right), p\left(=C / M r^{2}\right)$, are ${ }^{(1)}$ :

$$
\begin{gathered}
J_{2}=\frac{2}{3} e-\frac{1}{3} m \\
225 p^{2}-300 p+84+16\left[\left(\frac{15}{2} \cdot \frac{J_{2}}{e}-3\right) \cdot \frac{1}{1-\frac{4}{21} m}\right]=0
\end{gathered}
$$

where Eq. [1] holds even in the absence of hydrostatic equilibrium. Eliminating $e$ it follows that:

$$
p=\frac{2}{3}-\frac{4}{15} \sqrt{1-A} \quad ; A=\left(\frac{15}{2} \cdot \frac{J_{2}}{e}-3\right) /\left(1-\frac{4}{21} \cdot m\right)
$$

The obvious considerations follow from Eq. [3]; real $p$ and $p>0$ which imply:

$$
A \leqslant+1 \quad A \geqslant-21 / 4
$$

or

$$
J_{2} \leqslant 1.33 m \quad J_{2} \geqslant-3 / 39 m
$$


Rejecting the case where the moment of inertia with respect to an equatorial axis of the planet is greater than the moment of inertia with respect to the polar axis $(J<0)$, there will be a further limitation for $A$ which will have to be greater than -3 ; from this it follows that $p$ will have to be greater by about 0.13 , that is the possibility is excluded of an excessive concentration of mass at the centre.

\section{3. - Hydrostatid EQLiJibrium in THE SOLAR SYSTEM.}

For the purpose of investigating the planets in the solar system in the light of the considerations set out above, let us represent such planets in the $m, J_{2}$ plane. We note immediately that the points representing those planets whose fundamental parameters we know: mass, average radius, rotation period and $J_{2}$, belong with good approximation to a straight line that we shall call $\alpha$.

In this approximation one can obtain interesting information regarding the values of the moments of inertia of Uranus and Pluto and the mass of Pluto.

\section{4. - Moments of inertia of URANUs and PLUto.}

1st hypothesis: Let us suppose that the straight line must pass through the origin ( $p=$ const.), as well as through the points of the plane $m, J_{2}$ that represent the planets of which the mass, the radius, the angular rotation rate and the value of $J_{2}$ (Fig. 1) are known.

The value of "average" $p$ is found to be about 0.229 for all planets ( $J_{2} / m=$ const., approximating in the first order, imply $A=$ const.). The moments of inertia of Uranus is found to be: $C=1.31 \cdot 10^{47}$. For Pluto, using the observed values of $R=3200 \mathrm{~km}\left({ }^{3}\right)$, $M=6.59 \cdot 10^{26} \mathrm{gr}\left({ }^{8}\right)$, we obtain the value (1) of Table II.

2nd hypothesis: A straight line is plotted bearing in mind the known precision of the parameters of the various planets without foreing it to pass through the origin (Fig. 2).

For Uranus we obtain a value of $J_{2}$ equal to 0.0089 , a value of the dynamical flattening equal to 0.05 , in good agreement with the geometrical value derived from visual observations $\left(^{6}\right)$, a value of $p$ equal to 0.232 , and a moment of inertia $C=1.33 \cdot 10^{47} \mathrm{gr}^{2} \mathrm{~cm}^{2}$. 


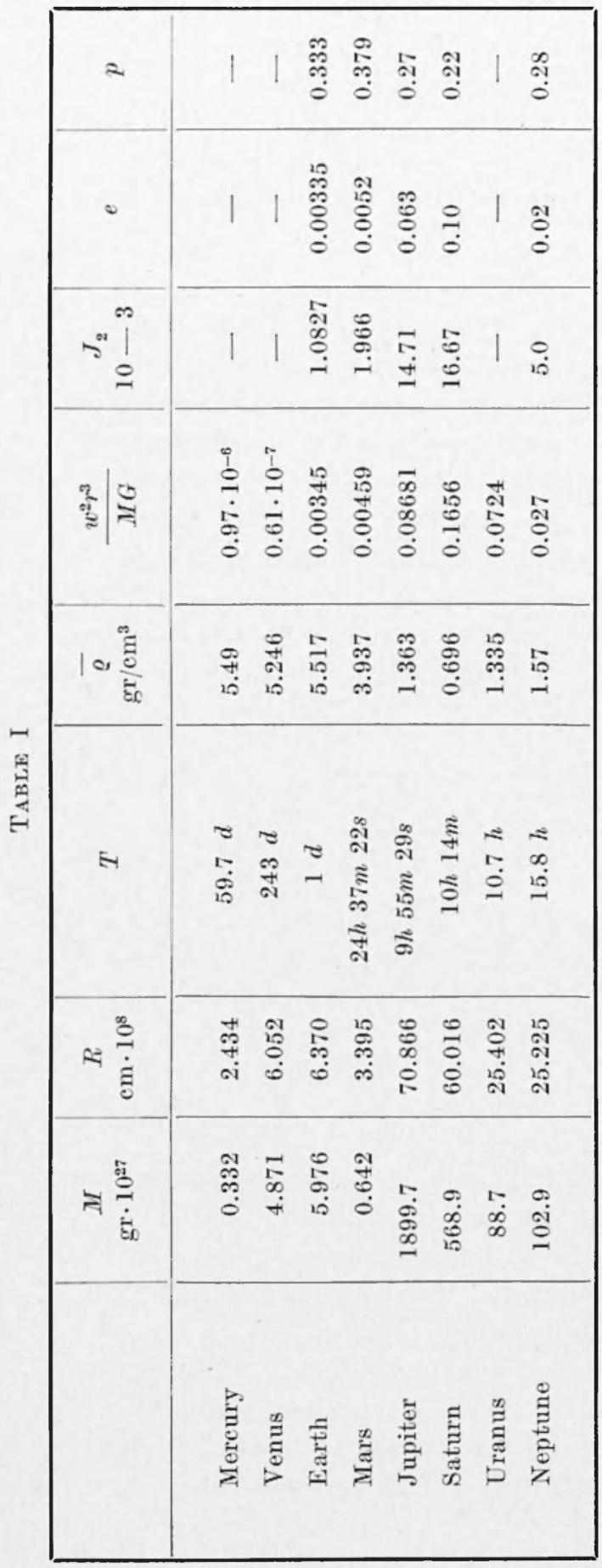

\begin{tabular}{|c|c|c|c|c|c|}
\hline$\frac{7}{3}$ & 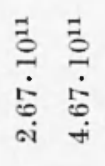 & $\begin{array}{l}\vec{\Xi} \\
\stackrel{0}{\dot{\alpha}} \\
\stackrel{0}{\circ}\end{array}$ & 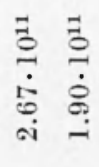 & $\begin{array}{l}\overrightarrow{0} \\
\stackrel{0}{\dot{1}} \\
\dot{0} \\
\text { i. }\end{array}$ & 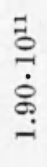 \\
\hline 0 & 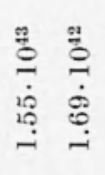 & $\begin{array}{l}\overrightarrow{0} \\
\dot{0} \\
\stackrel{\operatorname{c}}{1}\end{array}$ & 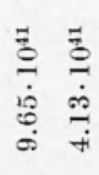 & 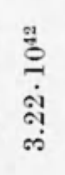 & 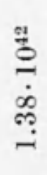 \\
\hline 2 & 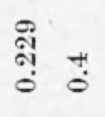 & $=$ & 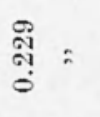 & $=$ & $=$ \\
\hline$\rightarrow$ & 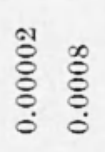 & $=$ & 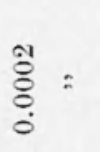 & $\begin{array}{l}\mathscr{8} \\
\delta \\
\delta \\
0\end{array}$ & $=$ \\
\hline$\approx$ & $\begin{array}{ll}\overrightarrow{0} & 0 \\
0 & 0 \\
0 & 0 \\
0 & 0\end{array}$ & $=$ & $=\quad \therefore$ & $\begin{array}{l}\text { श्ष } \\
\stackrel{0}{0} \\
\dot{0}\end{array}$ & : \\
\hline$\vec{z}$ & 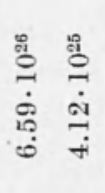 & 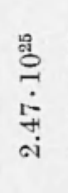 & 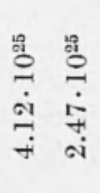 & ڤొ & $\begin{array}{l}\text { å } \\
\stackrel{+}{+} \\
\text { సั } \\
\infty\end{array}$ \\
\hline or & $\stackrel{\infty}{\stackrel{\infty}{+}} \stackrel{\dddot{0}}{0}$ & : & : & $\stackrel{\stackrel{i}{i}}{ }$ & $=$ \\
\hline Ret & $\begin{array}{ll}\stackrel{\infty}{0} & \stackrel{\infty}{!} \\
\dot{\sim} & \dot{\sim} \\
\oplus & \infty\end{array}$ & $\stackrel{\infty}{\stackrel{0}{\leftarrow}}$ & 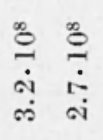 & $\begin{array}{l}\infty \\
\dot{0} \\
\dot{\sim}\end{array}$ & $\stackrel{\infty}{\stackrel{0}{7}}$ \\
\hline EN & 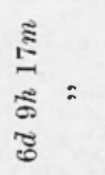 & $=$ & $=:=$ & : & $=$ \\
\hline & $-\infty$ & $\infty$ & Hi 10 & $\infty$ & to \\
\hline
\end{tabular}




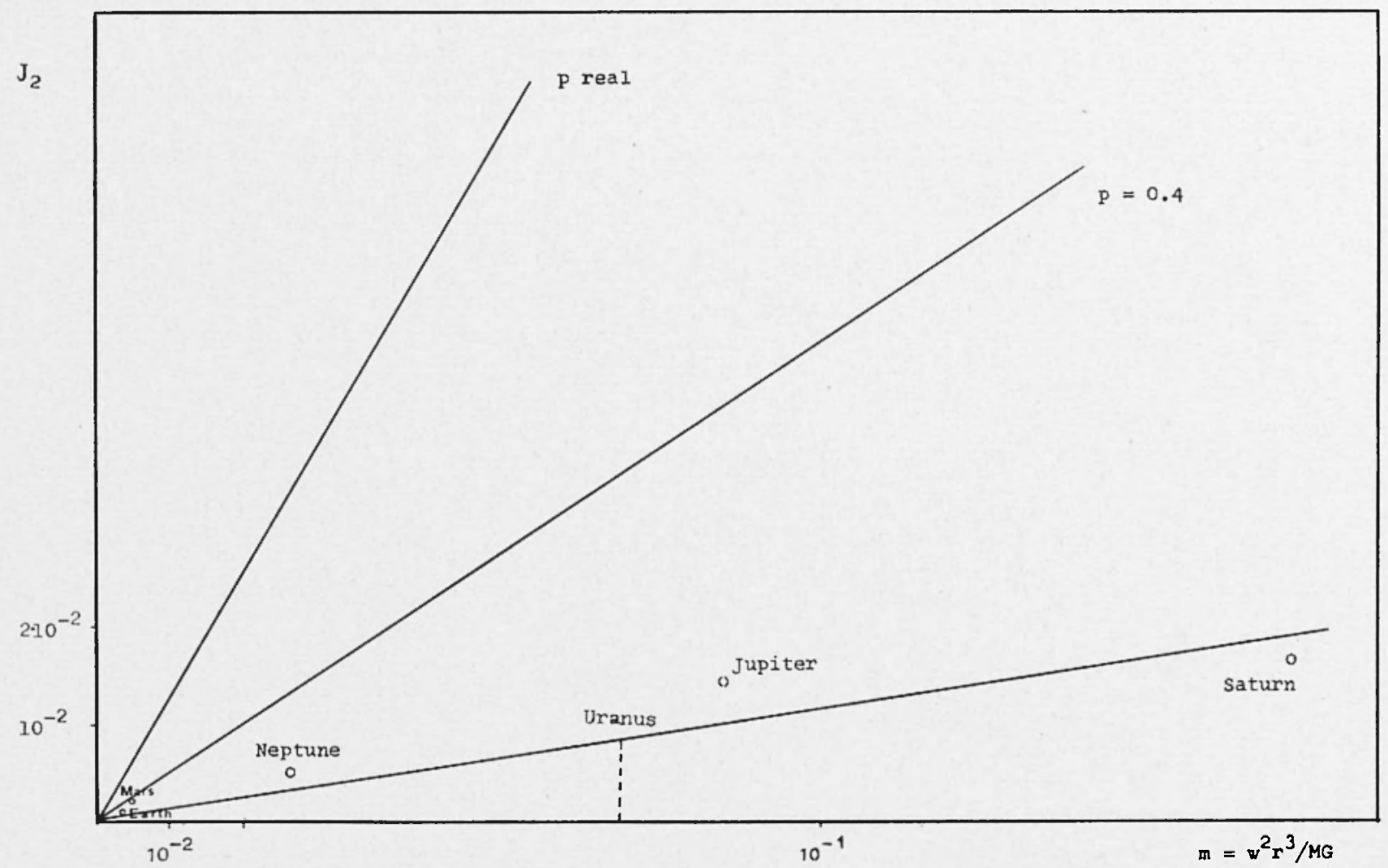

Fig. 1. - Representation of the planets in the plane $m=w-a^{3} / M G, J_{2}$; the linear regression approximating the points overall is made to pass through the origin. 


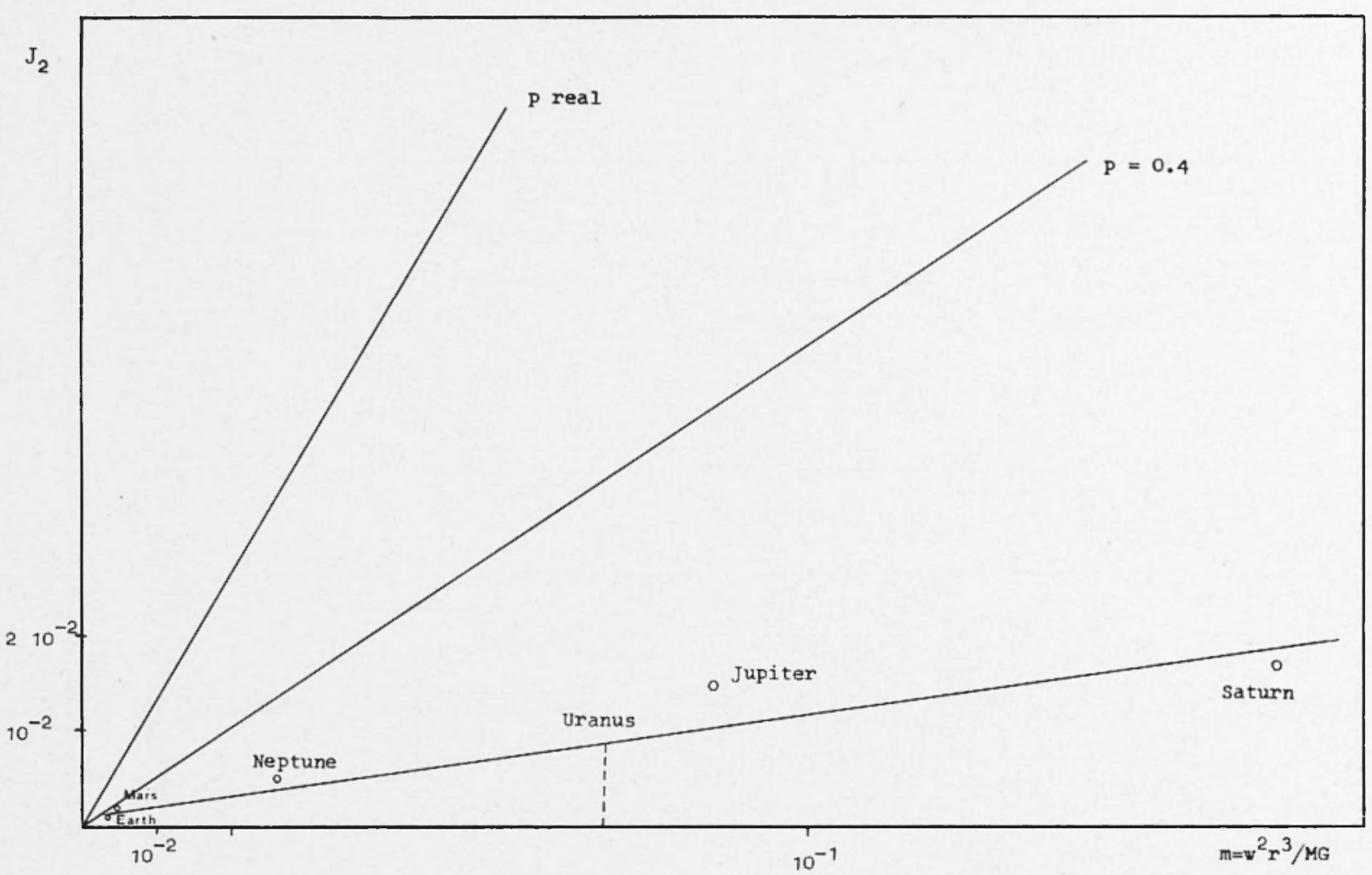

Fig. 2. - Representation of the planets in the plane $m=w^{2} a^{3} / M G, J_{2}$; the linear regression approximating the points overall is not made to pass through the origin. 
TIE HYDROSTATIC EQUILIBRIUM AND ITS IMPLICATIONS ETC.

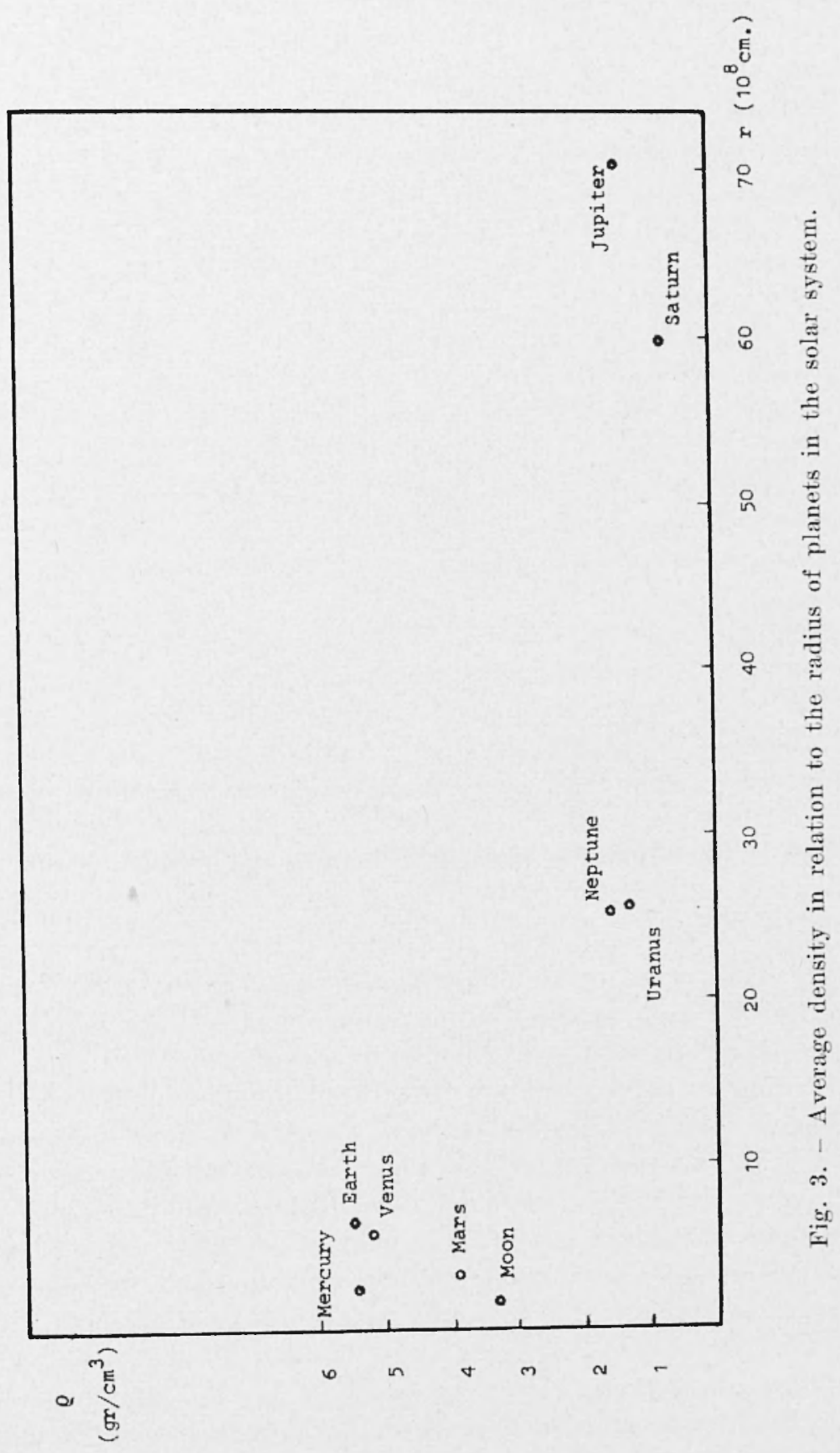




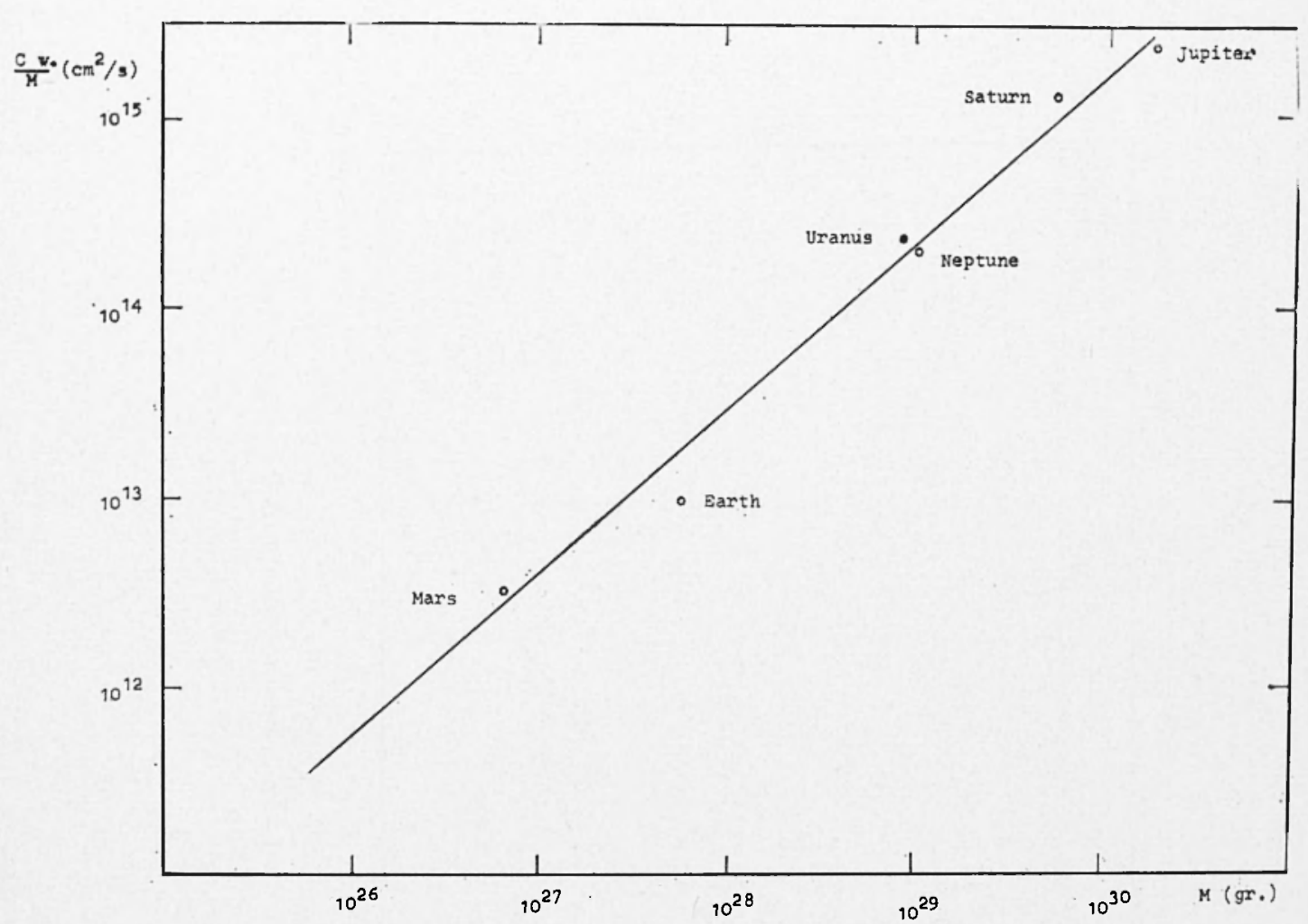

Fig. 4. - The density of the angular moment in relation to the mass.

For Pluto, making use of the values so far known and very uncertain of the mass and the radius, there would seem to be found a value of $p$ greater than 0.4 , which is unacceptable.

It must however be noted that the determination of the mass of Pluto is obtained from the study of the perturbations of Neptune, that is from the perturbations of a very small body on a very large one, and it is therefore extremely unreliable because a small error in the mass of Neptune causes a very large error in the mass of Pluto. A further difficulty arises from the fact that up to the present only a fraction of revolution of the outer planet has been observed. The best value of the mass obtained so far is $6.59 \cdot 10^{26} \mathrm{gr}\left({ }^{8}\right)$ and taking the radius of $3200 \mathrm{~km}\left({ }^{3}\right)$, one obtains an average density of 4.8 $\mathrm{gr} / \mathrm{cm}^{3}$, too high for an outer planet. Also we shall consirler the radius of Pluto $2700 \mathrm{~km}$ estimater from optical observation $\left({ }^{4}\right)$, although 
this radius, with the observed value of the mass gives an excessively high value of the mean density.

Considering now the average-mass density plane, one can observe that the planets take up positions along two lines: on one side we find terrestrial planets, on the other the outer planets (Fig. 3).

Pluto, an outer planet, should therefore belong to the straight line of outer planets and have an average density of about $2 \mathrm{gr} / \mathrm{cm}^{3}$ or less; accepting this hypothesis, there follows a diminution of the mass.

If we want to use the straight line which does not pass through the origin, a value of $p$ at most as large as 0.4 , and to keep the values of the radius, we obtain a value of $\varrho$ at most 0.3 and for $C$ the values indicated in lines (2) and (3) of Table II.

Using $\varrho=0.3$, the same values of $R$ and the straight line passing through the origin, we obtain the values of $C$ given in lines 4 and 5 of the same Table, while assuming $\varrho=2$ we obtain $C$ as in lines $(6)$ and $(7)$.

For a further check, we set out in Fig. 4 the values obtained by us of the "density of rotational angular momentum" $(C w / M)$ of Uranus, on the straight line obtained by MacDonald ( ${ }^{7}$ ) for planets whose moments of inertia are known with a fair degree of precision. The agreement of the data obtained here with those observed for other planets is satisfactory.

Obviously in Table II one finds that the values of the density of rotational angular momentum in lines (1), (4), (6) coincide as well as those of lines (5), (7); the difference of the two sets is due only on the different values of $R$ because the density of rotational angular momentum $C w / M$ can also be written pwR:. The values of $C w / M$ of line (1) is too far below the straight line of MacDonald; it would be difficult to explain the loss of the initial energy of the planet. The values of lines (2) and (3) are too far above and it would be difficult to explain the gain of energy. The only reasonable models of Pluto are those of lines (4), (5), (6) and (7).

\section{REFERENCES}

(1) Caputo, M., 1967. - The gravity field of the Earth. Acadenic I'ress, New lork.

(2) Соoк, A. H., 1972. - The dynamical properties and internal structures of the Earth, the Moon and the Planets. "Proc. R. soc.", Lond., A328, 301 . 
(3) Haldinay, I., Hardie, R. K., Franz, O. and Priser, J. B., 1966. "Publ. Astron. Soc. Pacific.", 78, 113.

(1) Haldinay, I., 1969. - "Publ. Astron. Soc. Pacific." 81, 285.

(5) KaULA, W. A., 1968. - An introduction to planetary physics. John Wiley Sons, Ine.

$\left({ }^{6}\right)$ Kovalevsky, J., 1970 . - Determination des masses des planètes et satel. lites. In (ed. A. Dollfus), "Surfaces and interiors of planets and satel. lites", 1-44, London, deadenic Press.

(i) MacDonaid, G. J. F., 1964. - Tidal friction. "Rev. Geoplyys.", 2, 467. 541 .

(8) Shinlimaxi, P. K., Klepczysseki, W. J., Duxcombe, R. L., 1971. Determination of the mass of Pluto. "Astron. J.", 76, 488. 